\title{
PROJETO DE REDES DE TRANSPORTE PÚBLICO POR ÔNIBUS: REVISÃO DE LITERATURA
}

\author{
Samille Cristina Rodrigues \\ Instituto Militar de Engenharia \\ Praça Gen. Tibúrcio, 80 - Urca, Rio de Janeiro - RJ \\ samillecr@gmail.com \\ Orivalde Soares da Silva Júnior \\ Instituto Militar de Engenharia \\ Praça Gen. Tibúrcio, 80 - Urca, Rio de Janeiro - RJ \\ orivalde@yahoo.com.br
}

\begin{abstract}
RESUMO
O presente artigo tem como objetivo apresentar uma revisão de literatura a respeito do projeto de redes de transporte público por ônibus (Transit Route Network Design Problem TRNDP), assim como suas várias definições, nomenclaturas, classificações, objetivos e metodologias usadas pelos diversos autores. Foram apresentadas as diferentes nomenclaturas dadas ao problema, suas características e etapas essenciais para o planejamento da rede, além dos métodos de solução mais usados na literatura. $\mathrm{O}$ artigo visa reunir as diversas definições utilizadas para o planejamento e desenho de rede, buscando contribuir com uma revisão sobre o assunto.
\end{abstract}

Palavras-chave: projeto de redes; transporte público por ônibus; planejamento de transportes.

\begin{abstract}
The present article aims to present a literature review of the transit route network design (TNDP), as well as its various definitions, nomenclatures, classifications, objectives and methodologies used by the various authors. We present the different nomenclatures given to the problem, its characteristics and essential stages for the planning of the network, besides the methods of solution most used in the literature. The article aims to compile the different definitions used for network planning and design, seeking to contribute to a review of the subject.
\end{abstract}

Keywords: network design; bus transit; transportation planning.

\section{Como Citar:}

RODRIGUES, Samille Cristina; SILVA JÚNIOR, Orivalde Soares da. PROJETO DE REDES DE TRANSPORTE PÚBLICO POR ÔNIBUS: REVISÃO DE LITERATURA. In: SIMPÓSIO DE PESQUISA OPERACIONAL E LOGÍSTICA DA MARINHA, 19. 2019, Rio de Janeiro, RJ. Anais [...]. Rio de Janeiro: Centro de Análises de Sistemas Navais, 2019. 


\section{INTRODUÇÃO}

A busca da mobilidade urbana e a melhoria dos sistemas de transportes públicos tem sido temas bastante discutidos quando se pensa em desenvolvimento e infraestrutura das cidades. Sabe-se que as políticas públicas voltadas para esse sistema são cada vez menos eficientes, dificultando o deslocamento das pessoas e a propagação do uso desse meio de transporte.

Além disso, o planejamento e design de uma rede de transporte público é altamente complexo, visto que dependem de vários fatores. Outro problema enfrentado pelo aumento do fluxo de transportes individuais nas vias, que são as emissões de gases poluentes e os custos ocasionados por acidentes de trânsitos e congestionamentos.

O planejamento do sistema de transporte público pode ser analisado através da demanda e oferta do serviço. A demanda surge da necessidade de deslocamento de um indivíduo acessar destinos espacialmente distribuídos em determinados períodos do dia. Ainda para um estudo de demanda de viagem, é preciso compreender a distribuição da demanda de acordo com a área de serviço e como ela se divide em diferentes estratos socioeconômicos (Muñoz e Giesen, 2010).

Já a oferta, é expressa pela disponibilidade de rotas para quem está disposto a viajar. Cada rota tem um ponto inicial e final específico, seguindo uma sequência de paradas. Visto que cada rota tem seu limite de passageiros, quanto maior a demanda menor o nível do serviço ofertado (Muñoz e Giesen, 2010).

Para Ceder e Wilson (1986), o planejamento da oferta de transporte público parte de uma decisão sistemática, onde cada etapa determina uma entrada para a próxima, assim como as decisões finais geram influência sobre as iniciais. Para Muñoz e Giesen (2010) o processo de planejamento de um transporte público pode ser estruturado em diferentes níveis de decisão, como estratégica, tática, operacional e de controle (Figura 1). 


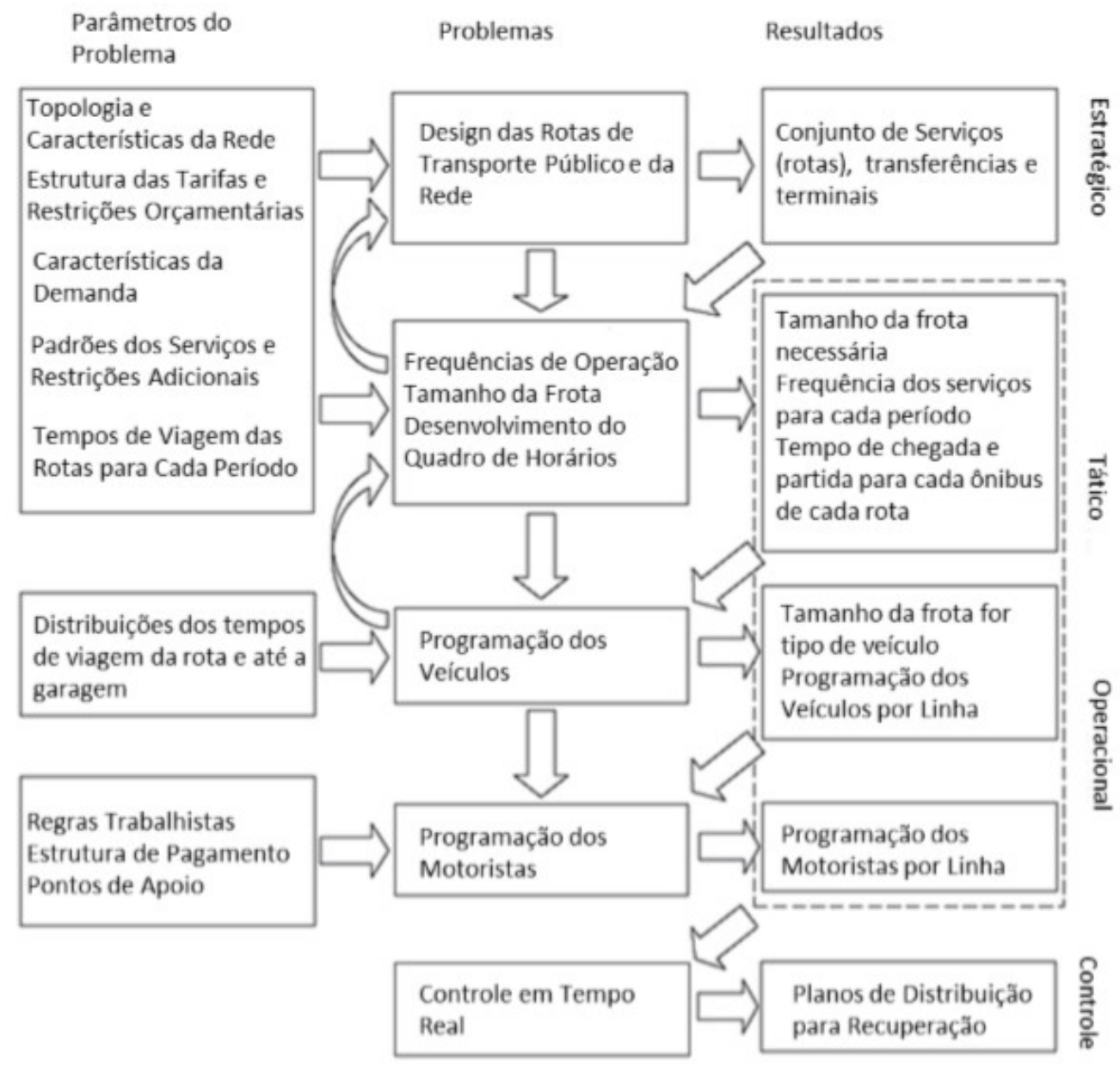

Figura 1: Processo de Planejamento da Operação de Ônibus

Fonte: Muñoz e Giesen (2010) adaptado de Ceder e Wilson (1986).

Nesse contexto, este artigo tem por finalidade apresentar as diferentes definições e nomenclaturas atribuídas ao projeto de redes de transporte público, assim como os objetivos, variáveis e restrições utilizadas no processo de solução. Identificando e revisando as diferentes metodologias do problema, assim como os critérios estabelecidos pelos diversos autores.

Após esta introdução, a seção 2 apresenta os problemas mais utilizados na revisão de literatura, assim como as diferentes características adotadas. A seção 3 trata das etapas previstas para a resolução do problema apresentado e as principais metodologias aplicadas aos modelos matemáticos propostos. A seção 4 exemplifica os estudos de caso aplicados a redes reais e por fim a conclusão. 


\section{PROBLEMAS UTILIZADAS NA REVISÃO DE LITERATURA}

Para o Projeto de Redes de Transporte Público (PRTP), o termo mais utilizado na literatura se dá por “transit route network design”. Para a palavra design traduziu-se como projeto, network para rede, sendo o conjunto de vias e acessos utilizadas para o serviço de transporte. Route se traduz como rota ou linha de ônibus e transit sendo o transporte coletivo de passageiros, sendo traduzido para transporte público (Arbex, 2015).

Para Faharani et. al. (2013), o Projeto de Redes de Transporte Público se encaixa no Urban Transportation Network Design Problem (UTNDP). Em sua revisão sobre o assunto, o autor apresenta as definições, classificações, objetivos, restrições e métodos utilizados na resolução do problema, onde se encaixam tanto o Public Transit Network Design Problem (PTNDP) que trata do problema do projeto da rede do transporte público, e Road Network Design Problem (RNDP) que é o problema sobre o projeto de rede do sistema viário, não distinguindo entre transporte público e outros veículos particulares.

Para Fan e Machemehl (2004), o problema em que rotas e frequências de trânsito são definidas se dá por Bus Transit Route Network Design Problem (BTRNDP), onde é considerada uma demanda de tráfego variável. Guihaire e Hao (2008) classificam o problema de acordo com as etapas do processo de planejamento do transporte público proposto por Ceder e Wilson (1986). As cinco etapas são: projeto da rede (network design) atribuição de frequências, elaboração do quadro de horários de partidas, agendamento dos ônibus e agendamento dos motoristas.

No entanto, para obter uma melhor qualidade nos resultados, é importante que todas as etapas sejam tratadas simultaneamente garantindo a interação e o feedback. Mas devido à complexidade do problema, essa abordagem global ainda é intratável na prática. Até mesmo os subproblemas tratados separadamente são considerados NP-hard do ponto de vista computacional (Guihaire e Hao, 2008). Diante disto foram consideradas apenas as três primeiras etapas (projeto de rede, atribuição de frequências e quadro de horários) proposta na classificação dos autores. A classificação proposta por Guihaire e Hao (2008) segue na Figura 2. 


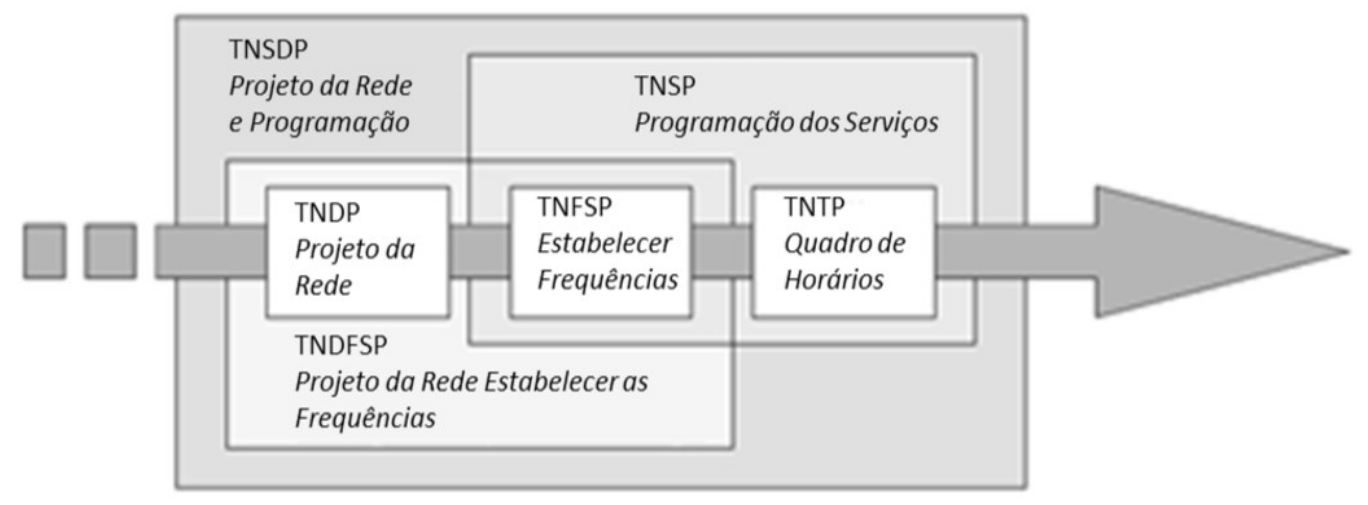

Figura 2: Classificação e Nomenclatura dos problemas correlatos ao PRTP.

Fonte: Arbex (2015)

Kepaptsoglou e Karlaftis (2009) também trataram em sua revisão de literatura do problema do projeto de rotas da rede de transporte público, que o autor nomeou de Transit Route Network Design Problem (TRNDP), mas não especificando se o trabalho também calcula a frequência.

\section{REVISÃO DAS ETAPAS PARA SOLUÇÃO DO PROBLEMA}

O problema de Projeto de Redes de Transporte Público é caracterizado como um problema de otimização onde a função objetivo é definida, as restrições são determinadas e a metodologia utilizada é escolhida para obter um projeto de rede ideal (Kepaptsoglou e Karlaftis, 2009). Nesta seção serão abordados os principais métodos de solução utilizados pelos autores, assim como definição da função objetivo, restrições utilizadas, dados de entrada, variáveis de decisão e principais heurísticas abordadas para resolução do problema.

\subsection{Função Objetivo}

Em sua maioria, as revisões de literatura consideram a função objetivo como multiobjetivo, já que o problema trata de minimizar tanto os custos dos usuários do transporte como os custos dos operadores (Mandl, 1980; Pattnaik et al., 1998).

Por se tratar de um problema multiobjetivo e complexo, torna-se de difícil resolução sua aplicação usando os métodos tradicionais de programação matemática, tornando-os ineficientes (Baaj and Mahmassani, 1991). A Tabela 1 lista alguns autores e suas respectivas funções objetivo. 
Tabela 1. PRTP Função Objetivo

\begin{tabular}{|c|c|c|}
\hline Ano & Autor & Função Objetivo \\
\hline 1980 & Mandl & Custo do transporte \\
\hline 1986 & Ceder e Wilson & Custos de operação e Usuários \\
\hline 1991 & Baaj e Mahmassani & $\begin{array}{l}\text { Tempo total de viagem, } \\
\text { número de transferências, } \\
\text { número de ônibus }\end{array}$ \\
\hline 1998 & Pattnaike et. al & Custos de operação e Usuários \\
\hline 2004 & Petrelli & Custos de operação e Usuários \\
\hline 2006 & Fan e Machemehl & Custos de operação e Usuários \\
\hline 2007 & Zhao e Zeng & Custos de operação e Usuários \\
\hline
\end{tabular}

Fonte: Autor (2019) adaptado de Kepatsoglou e Karlaftis (2009)

\subsection{Restrições}

Para os modelos matemáticos utilizados na literatura são consideradas restrições que especificam o que uma rede real deve atender, de tal forma a encontrar soluções que sejam viáveis de serem aplicadas às cidades (Arbex, 2015).

Fan e Machemehl (2006) caracterizam as restrições como o reflexo do desempenho da rede de transporte público juntamente com suas limitações de recursos. Alguns tipos de restrições são listadas por Zhao and Zeng (2006) como: viabilidade das frequências, fatores de carga máxima e mínima (load fator), o formato, direção, comprimento máximo e número de rotas, tamanho da frota e custos operacionais.

Kepaptsoglou e Karlaftis (2009) consideram as restrições como: restrições de desempenho e restrições de recursos. Nas restrições de desempenho se encaixam: viabilidade de operação das frequências de cada linha da rede, limite de lotação dos veículos, extensão máxima e mínima da rota, itinerário da rota, área de cobertura, ligações diretas promovidas pelas rotas e atendimento da demanda.

No que diz respeito às restrições de recursos estão aquelas em que correspondem ao tamanho da frota e orçamento da mesma (Kepaptsoglou e Karlaftis, 2009). É importante salientar que a frota é um dos principais componentes dos custos dos operadores, visto que a maioria dos trabalhos revisados buscam a minimização das frotas juntamente com os custos dos usuários (Arbex, 2015).

Guihaire e Hao (2008) apresentam outras restrições que julgam ser importantes para o planejamento do projeto de redes do transporte público. São elas: histórico, número de linhas, objetivos específicos do operador, o nós devem ser únicos na rota, mas podem se 
repetir na rede, todos os nós da rede devem ser atendidos por pelo menos uma rota (Figura 3), as rotas devem estar conectadas entre si e a mesma rota não pode ser repetida em uma rede.

Todas essas restrições são importantes para o bom funcionamento do modelo matemático proposto, proporcionando assim soluções de rede que atendam as particularidades e a qualidade do transporte público.

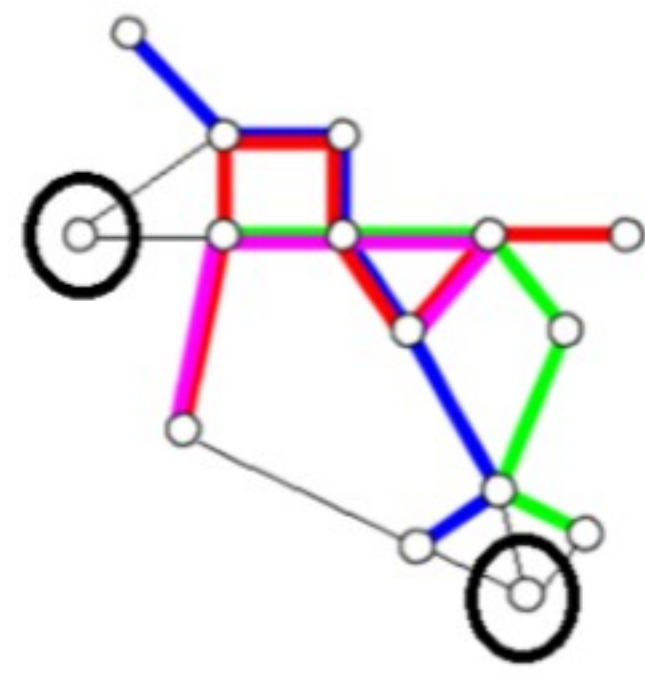

Figura 3: Rede inviável, pois nem todos os nós foram atendidos.

Fonte: Arbex (2015)

\subsection{Variáveis de Decisão}

Nos trabalhos revisados as variáveis de decisão comumente aplicadas são o traçado das rotas e frequências de operação da rede. Alguns autores também consideram as tarifas a serem cobradas, os pontos de paradas e os tipos de ônibus adotados em cada linha.

Para obter um melhor desempenho dos métodos de solução, todos os modelos utilizados deveriam considerar as cinco variáveis de decisão. Todavia, em virtude da complexidade do problema ao considerar todas as variáveis, os trabalhos revisados utilizaram apenas o traçado das rotas e a frequência de operação (Arbex, 2015).

\subsection{Dados de entrada}

Os dados de entrada mais utilizados nos trabalhos revisados são a demanda de transporte público, demanda de transporte individual, tempo de viagem na rede, sistema viário e rede de transporte público já existente. 
Para os trabalhos que consideraram a demanda variável, foram inseridos também, além da demanda de transporte público, a demanda de transporte individual, visto que o modelo proposto parte de que uma demanda total de transporte pode ser atraída para o transporte público, sendo necessário o somatório das duas no modelo.

Para modelos mais complexos em que a rede de transporte existente é dado de entrada, devem ser consideradas todos os sistemas já implantados, como corredores de ônibus e sistemas metro-ferroviários.

\subsection{Metodologias aplicadas}

Devido à complexidade dos problemas de planejamento de projetos de redes de transporte público, o uso de formulações de programação matemática tradicionais não podem ser aplicados para garantir um resultado eficiente. Para Baaj e Mahmassani (1991), devido a sua natureza combinatória o problema de PRTP acaba se tornando NP-hard, de difícil resolução.

Por ser um problema multiobjetivo e complexo, é difícil encontrar uma solução formal de otimização exata para o problema. Levando isso em consideração, a maioria dos autores revisados usam métodos heurísticos para solucionar o problema. Faharani et. al (2013) classificam os métodos de solução em três categorias: (1) métodos matemáticos exatos, (2) heurísticas e (3) meta-heurísticas. Segundo os autores, métodos matemáticos exatos como o branch-and-bound são inaplicáveis para redes de tamanho médio e grande, visto a sua ineficiência computacional. Já as heurísticas são mais eficientes que os métodos exatos, podendo ser aplicado a redes de tamanhos maiores.

Meta-heurísticas como Simulated Annealing (AS) e Algoritmo Genético (GA) são baseados em processos físicos, químicos ou biológicos. Estes não necessitam de nenhuma garantia matemática de que atingiram a solução ótima, sendo usados para boas soluções. Quanto à velocidade computacional, as meta-heurísticas possuem uma velocidade maior do que os métodos exatos. A Figura 4 mostra as principais meta-heurísticas aplicadas aos problemas de projeto de redes de transporte público, sendo Algoritmo Genético (GA), Recozimento Simulado (AS), Busca Tabu (TS) e Colônia de Formigas (AC). 


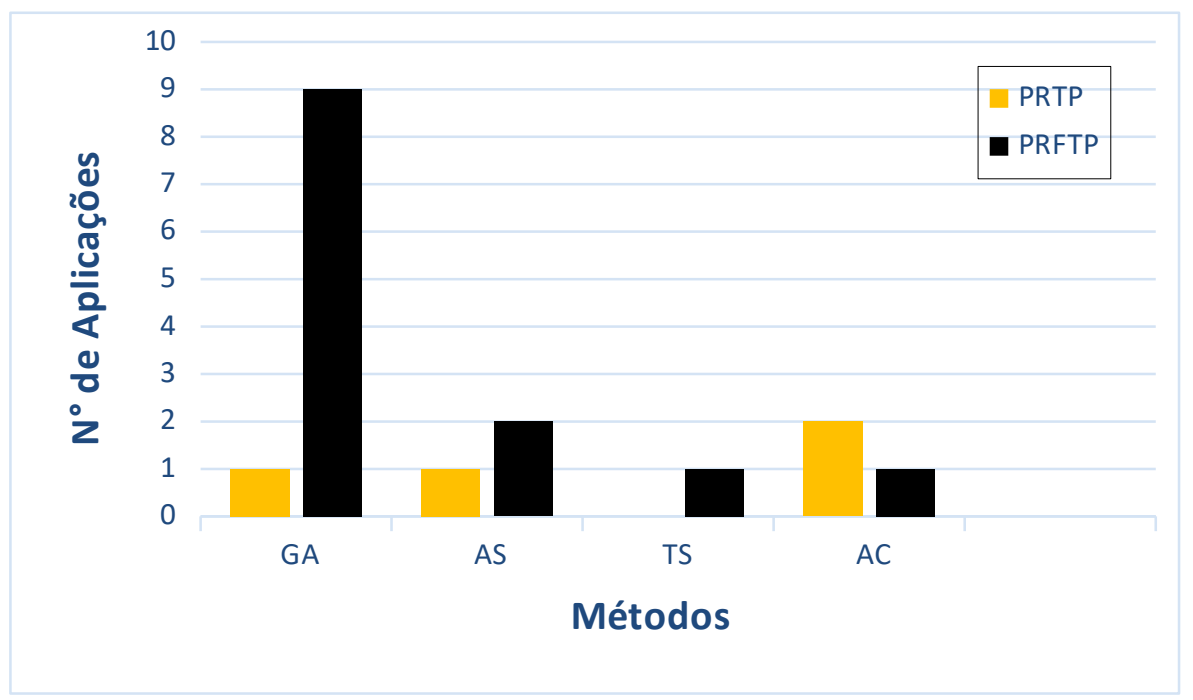

Figura 4: Principais meta-heurísticas aplicadas ao UTNDP.

Fonte: Autor (2019) adaptado de Faharani et.al (2013).

Kepaptsoglou e Karlaftis (2009) caracterizam os métodos heurísticos em dois grupos. O primeiro contém o problema da geração de um banco de rotas inicial contendo rotas fixas e únicas e posteriormente a criação de um subgrupo deste banco de rotas. No segundo grupo se encaixa a construção do banco de rotas através de heurísticas ou meta-heurísticas diretamente, e se necessário à melhoria do conjunto de rotas.

Para criação do banco de rotas, que são geradas no início do processo, geralmente são usados algoritmos de caminho mínimo, gerando as rotas segundo as restrições anteriormente definidas. Fan e Machemmehl (2006) usaram algoritmos de caminho mínimo como de Dijkstra e Yen para a geração do banco de rotas.

$\mathrm{Na}$ criação e melhoria das rotas, meta-heurísticas podem ser aplicadas diretamente para construção do banco de rotas e posterior determinação da frequência. Ceder e Wilson (1986) utilizaram um método de dois níveis para a configuração das rotas. O primeiro nível se baseava apenas na perspectiva do usuário, sendo utilizado um algoritmo de otimização para selecionar as rotas ótimas. No segundo nível foi aplicada uma heurística em que eram consideradas tanto a perspectiva do usuário como a do motorista para determinar as rotas e as frequências.

No que diz respeito à alocação da demanda à rede proposta, poucos autores mencionaram essa parte nos seus trabalhos. 


\section{ESTUDOS DE CASOS}

Por se tratar de um problema real e prático, alguns autores revisados aplicaram seus modelos desenvolvidos em cidades reais, contribuindo assim para fornecer informações a respeito do tamanho da rede para cada problema tratado futuramente. A Tabela 2 lista os modelos de projeto de redes de transporte públicos que já foram aplicados a casos reais.

Tabela 2. Estudos de casos para PRTP e PRFTP

\begin{tabular}{|c|c|c|c|}
\hline Problema & Referência & Cidade, País & Tamanho da rede \\
\hline \multirow{6}{*}{$\begin{array}{l}\text { Problema de Projeto } \\
\text { de Rede de } \\
\text { Transporte Público }\end{array}$} & Mandl (1980) & Rede de Swiss & 14 nós e $20 \operatorname{arcos}$ \\
\hline & Zhao e Zang (2006) & Miami, USA & $\begin{array}{r}4300 \text { ruas, } 2804 \text { nós e } \\
120.000 \text { pares O/D }\end{array}$ \\
\hline & $\begin{array}{l}\text { Yu et al. (2005) and } \\
\text { Yang et al. (2007) }\end{array}$ & Dalian, China & $\begin{array}{l}3200 \text { arcos, } 2300 \text { nós e } \\
1500 \text { pontos de paradas }\end{array}$ \\
\hline & Guan et al. (2006) & Hong Kong & 9 nós e 36 pares $\mathrm{O} / \mathrm{D}$ \\
\hline & $\begin{array}{r}\text { Mauttone and } \\
\text { Urquhart (2009) }\end{array}$ & Riviera, Uruguai & 84 nós e 143 arcos \\
\hline & $\begin{array}{r}\text { Curtin and Biba } \\
(2011)\end{array}$ & Richardson, Texas & Não há dados \\
\hline \multirow{4}{*}{$\begin{array}{l}\text { Problema de Projeto } \\
\text { de Rede e } \\
\text { Frequências de } \\
\text { Transporte Público }\end{array}$} & Dubois et. al & $\begin{array}{r}\text { Dez cidades da } \\
\text { França }\end{array}$ & $\begin{array}{r}\text { Niece: } 700 \text { nós, } 250 \\
\text { zonas e } 5400 \operatorname{arcos}\end{array}$ \\
\hline & $\begin{array}{r}\text { Shih et al. (1998) } \\
\text { and Baaj and } \\
\text { Mahmassani (1995) }\end{array}$ & Austin, USA & 177 pontos de paradas \\
\hline & $\begin{array}{r}\text { Pattnaik et al. } \\
\text { (1998) }\end{array}$ & $\begin{array}{l}\text { Uma parte de } \\
\text { Madras, Índia }\end{array}$ & 25 nós e 35 arcos \\
\hline & $\begin{array}{r}\text { Pacheco et al. } \\
\text { (2009) }\end{array}$ & Burgos, Espanha & 382 pontos de paradas \\
\hline
\end{tabular}

Fonte: Autor (2019) adaptado de Kepatsoglou e Karlaftis (2009)

Em sua revisão de literatura Farahni et. al (2013) concluiu que a maioria dos problemas de projetos de rede de transporte não tem aplicação no mundo real. Segundo os autores, grande parte dos problemas estudados tem como foco apenas o desenvolvimento de métodos de soluções eficientes para problemas práticos, visto que em sua maioria, os autores não possuem dados reais para aplicações dos métodos. 


\section{CONCLUSÃO}

O presente artigo teve como objetivo fazer uma análise dos diversos estudos aplicados ao projeto de redes de transporte público por ônibus. Foi realizada uma revisão da literatura sobre o assunto, indicando as diversas nomenclaturas dadas ao problema, assim como os diversos e principais métodos de solução utilizados pelos autores.

Foi possível observar que se trata de um problema bastante complexo e de difícil solução por métodos exatos, sendo necessária a aplicação de métodos meta-heurísticos como algoritmos evolutivos e heurísticas específicas para resolver o problema estudado. Além do mais, uma importante análise dos critérios para elaboração do modelo matemático como função objetivo, variáveis de decisão e restrições, são necessários para a obtenção de uma solução adequada.

Quanto aos estudos de caso, observou-se que poucos deles realizam estudos de caso com redes reais e de grande porte, utilizando-se geralmente de problemas fictícios e reduzidos. Observa-se que a coleta de dados reais ainda é uma parte de difícil acesso para os autores. Foi possível observar também que o planejamento da rede para eventuais casos de emergências ou eventos específicos não tem sido estudado e sendo uma lacuna identificada no projeto de redes de transporte público por ônibus.

\section{REFERÊNCIAS BIBLIOGRÁFICAS}

[1] Afandizadeh, Sh, H Khaksar, e N Kalantari. "Bus Fleet Optimization Using Genetic Algorithm a Case Study of Mashhad”. International Journal of Civil Engineering 11, $\mathrm{n}^{\mathrm{o}} 1$ ([s.d.]): 10 .

[2] Agrawal, Jitendra, e Tom V. Mathew. "Transit Route Network Design Using Parallel Genetic Algorithm". Journal of Computing in Civil Engineering 18, $\mathrm{n}^{\circ} 3$ (julho de 2004): 248-56. https://doi.org/10.1061/(ASCE)0887-3801(2004)18:3(248).

[3] Arbex, Renato Oliveira. "Projeto de redes otimizadas de transporte público por ônibus utilizando algoritmo genético." Mestrado em Engenharia de Transportes, Universidade de São Paulo, 2014. https://doi.org/10.11606/D.3.2014.tde-16112015-160850.

[4] Baaj, M. Hadi, e Hani S. Mahmassani. "An AI-Based Approach for Transit Route System Planning and Design". Journal of Advanced Transportation 25, $\mathrm{n}^{\circ} 2$ (março de 1991): 187-209. https://doi.org/10.1002/atr.5670250205.

[5] Bielli, Maurizio, Massimiliano Caramia, e Pasquale Carotenuto. "Genetic Algorithms in Bus Network Optimizationq", 2002, 16.

[6] Cancela, Héctor, Antonio Mauttone, e María E. Urquhart. "Mathematical Programming Formulations for Transit Network Design". Transportation Research Part B: Methodological 77 (julho de 2015): 17-37. https://doi.org/10.1016/j.trb.2015.03.006. 
[7] Carlos Muñoz, Juan, e Ricardo Giesen. "Optimization of Public Transportation Systems". In Wiley Encyclopedia of Operations Research and Management Science, por James J. Cochran, Louis A. Cox, Pinar Keskinocak, Jeffrey P. Kharoufeh, e J. Cole Smith, eorms0935. Hoboken, NJ, USA: John Wiley \& Sons, Inc., 2011. https://doi.org/ 10.1002/9780470400531.eorms0935.

[8] Ceder, Avishai, e Nigel H.M. Wilson. "Bus Network Design". Transportation Research Part B: Methodological 20, $\mathrm{n}^{\circ} 4$ (agosto de 1986): 331-44. https://doi.org/ 10.1016/0191-2615(86)90047-0.

[9] Chakroborty, Partha. "Genetic Algorithms for Optimal Urban Transit Network Design". Computer-Aided Civil and Infrastructure Engineering 18, $\mathrm{n}^{\circ} 3$ (maio de 2003): 184-200. https://doi.org/10.1111/1467-8667.00309.

[10] Chakroborty, Partha, e Tathagat Wivedi. "Optimal Route Network Design for Transit Systems Using Genetic Algorithms". Engineering Optimization 34, $\mathrm{n}^{\circ} 1$ (janeiro de 2002): 83-100. https://doi.org/10.1080/03052150210909.

[11] Chew, Joanne Suk Chun, e Lai Soon Lee. "A GENETIC ALGORITHM FOR URBAN TRANSIT ROUTING PROBLEM". International Journal of Modern Physics: Conference Series 09 (janeiro de 2012): 411-21. https://doi.org/10.1142/ S2010194512005491.

[12] Fan, Wei, e Randy B. Machemehl. "Optimal Transit Route Network Design Problem with Variable Transit Demand: Genetic Algorithm Approach”. Journal of Transportation Engineering 132, $\mathrm{n}^{\circ} 1$ (janeiro de 2006): 40-51. https://doi.org/10.1061/ (ASCE)0733-947X(2006)132:1(40).

[13] Farahani, Reza Zanjirani, Elnaz Miandoabchi, W.Y. Szeto, e Hannaneh Rashidi. "A Review of Urban Transportation Network Design Problems". European Journal of Operational Research 229, nº 2 (setembro de 2013): 281-302. https://doi.org/10.1016/ j.ejor.2013.01.001.

[14] - "A Review of Urban Transportation Network Design Problems". European Journal of Operational Research 229, n 2 (setembro de 2013): 281-302. https:// doi.org/10.1016/j.ejor.2013.01.001.

[15] Guihaire, Valérie, e Jin-Kao Hao. "Transit Network Design and Scheduling: A Global Review". Transportation Research Part A: Policy and Practice 42, $\mathrm{n}^{\circ} 10$ (dezembro de 2008): 1251-73. https://doi.org/10.1016/j.tra.2008.03.011.

[16] Jha, Shashi Bhushan, J.K. Jha, e Manoj Kumar Tiwari. "A Multi-Objective MetaHeuristic Approach for Transit Network Design and Frequency Setting Problem in a Bus Transit System". Computers \& Industrial Engineering 130 (abril de 2019): 16686. https://doi.org/10.1016/j.cie.2019.02.025.

[17] Kepaptsoglou, Konstantinos, e Matthew Karlaftis. "Transit Route Network Design Problem: Review". Journal of Transportation Engineering 135, n 8 (agosto de 2009): 491-505. https://doi.org/10.1061/(ASCE)0733-947X(2009)135:8(491).

[18] Kiliç, Fatih, e Mustafa Gök. "A Public Transit Network Route Generation Algorithm". IFAC Proceedings Volumes 46, $\mathrm{n}^{\mathrm{o}} 25$ (2013): 162-66. https://doi.org/ 10.3182/20130916-2-TR-4042.00033.

[19] M, Kalochristianakis, e Kosmopoulos D. "A Study and Implementation of the Transit Route Network Design Problem for a Realisitic Urban Case”. In Computer Science \& Information Technology ( CS \& IT ), 59-65. Academy \& Industry Research Collaboration Center (AIRCC), 2015. https://doi.org/10.5121/csit.2015.51506. 
[20] Mandl, Christoph E. "Evaluation and Opt "mi Zation of Urban Public Transportation Networks", [s.d.], 9.

[21] Mauttone, Antonio, e María E. Urquhart. "A Route Set Construction Algorithm for the Transit Network Design Problem”. Computers \& Operations Research 36, $\mathrm{n}^{\circ} 8$ (agosto de 2009): 2440-49. https://doi.org/10.1016/j.cor.2008.09.014.

[22] Pattnaik, S. B., S. Mohan, e V. M. Tom. "Urban Bus Transit Route Network Design Using Genetic Algorithm". Journal of Transportation Engineering 124, n 4 (julho de 1998): 368-75. https://doi.org/10.1061/(ASCE)0733-947X(1998)124:4(368).

[23] Petrelli, M. "A Transit Network Design Model for Urban Areas". Urban Transport X, 2004, 10.

[24] Sadrsadat, Hadi, Hossein Poorzahedi, Ali Haghani, e Elham Sharifi. "Bus Network Design Using Genetic Algorithm", [s.d.], 16.

[25] Srinivas, M., e L.M. Patnaik. "Genetic Algorithms: A Survey”. Computer 27, nº 6 (junho de 1994): 17-26. https://doi.org/10.1109/2.294849.

[26] Yan, Yadan, Zhiyuan Liu, Qiang Meng, e Yu Jiang. "Robust Optimization Model of Bus Transit Network Design with Stochastic Travel Time". Journal of Transportation Engineering 139, $\mathrm{n}^{\circ} 6$ (junho de 2013): 625-34. https://doi.org/10.1061/ (ASCE)TE.1943-5436.0000536.

[27] Yu, Bin, Chuntian Cheng, Zhongzhen Yang, e Chong Liu. "OPTIMIZING BUS TRANSIT NETWORK WITH PARALLEL ANT COLONY ALGORITHM”. Proceedings of the Eastern Asia Society for Transportation Studies 5 (2005): 16.

[28] Zhao, F., e X. Zeng. "Optimization of Transit Network Layout and Headway with a Combined Genetic Algorithm and Simulated Annealing Method". Engineering Optimization 38, $\mathrm{n}^{\mathrm{o}} 6$ (setembro de 2006): 701-22. https://doi.org/ $\underline{10.1080 / 03052150600608917 .}$. 